\title{
CANONICAL QUOTIENT SINGULARITIES IN DIMENSION THREE
}

\author{
DAVID R. MORRISON ${ }^{1}$
}

\begin{abstract}
We classify isolated canonical cyclic quotient singularities in dimension three, showing that, with two exceptions, they are all either Gorenstein or terminal. The proof uses the solution of a combinatorial problem which arose in the study of algebraic cycles on Fermat varieties.
\end{abstract}

This note is a continuation of our previous paper [7] (written jointly with Glenn Stevens), which studied certain canonical quotient singularities in dimensions three and four. The classification of canonical quotient singularities can be reduced to a certain combinatorial problem, which we partially solved in [7]. After [7] went to press, we learned that a similar (although not quite identical) combinatorial problem occurs in the study of algebraic cycles on Fermat varieties (cf. $[6,8,10])$. In this note, we apply the recent solution of that problem (due to Aoki and Shioda $[1,2,10]$ ) to the classification of canonical quotient singularities in dimension three.

If $x$ is a rational number, we let $\langle x\rangle$ denote the rational number such that $x \equiv\langle x\rangle \bmod \mathbf{Z}$, and $0 \leqslant\langle x\rangle<1$.

DEFINITION. A Fermat quadruple is a quadruple of rational numbers $\left(a_{1} / N, a_{2} / N, a_{3} / N, a_{4} / N\right)$ such that $a_{i} \not \equiv 0(\bmod N)$, g.c.d. $\left\{a_{i}\right\}=1$, and, for every integer $k$ with $(k, N)=1, \Sigma_{i}\left\langle a_{i} k / N\right\rangle=2$.

The following theorem was conjectured by Meyer and Neutsch [6] and Shioda $[10]$, and proved by Aoki and Shioda $[1,2,10]$.

THEOREM 1. Let $\left(a_{1} / N, a_{2} / N, a_{3} / N, a_{4} / N\right)$ be a Fermat quadruple. Then there is some $k$ with $(k, N)=1$ such that $\left(\left\langle a_{1} k / N\right\rangle,\left\langle a_{2} k / N\right\rangle,\left\langle a_{3} k / N\right\rangle,\left\langle a_{4} k / N\right\rangle\right)$ is, after reordering, one of the following:

(i) $(\alpha / N, 1-(\alpha / N), \beta / N, 1-(\beta / N))$ for some $0<\alpha, \beta<N$,

(ii) $(1 / 2 K, K / 2 K,(K+1) / 2 K,(2 K-2) / 2 K)$ where $N=2 K$,

(iii) $(1 / 2 K,(K+1) / 2 K,(K+2) / 2 K,(2 K-4) / 2 K)$ where $N=2 K$,

(iv) $(1 / 3 K,(K+1) / 3 K,(2 K+1) / 3 K,(3 K-3) / 3 K)$ where $N=3 K$,

(v) one of 101 "exceptional quadruples" listed in [6]: each has $(N, 6) \neq 1$ and $N \leqslant 180$.

Received by the editors February 9, 1984.

1980 Mathematics Subject Classification. Primary 14B05; Secondary 14L30, 32B30.

Key words and phrases. Canonical singularity, Fermat variety, Gorenstein ring, quotient singularity, terminal singularity.

${ }^{1}$ National Science Foundation Postdoctoral Fellow. 
The combinatorial fact which we used in [7] to classify terminal quotient singularities is

THEOREM 2 [13, 3, 4 AND 7]. Let $a, b, c$ be integrers relatively prime to $N$. Suppose, for all $k \in \mathbf{Z}-N \mathbf{Z}$,

$$
\langle a k / N\rangle+\langle b k / N\rangle+\langle c k / N\rangle>1 .
$$

Then, after reordering $a, b, c$, we have $a+b \equiv 0(\bmod N)$.

Note that under the hypotheses of Theorem 2, if we let $d=-(a+b+c)$, then $(a / N, b / N, c / N, d / N)$ is a Fermat quadruple. For $N$ prime, Theorems 1 and 2 are equivalent; this case of these theorems was also proved by W. Parry (cf. [6 and 8]).

We can now state our main combinatorial result.

THEOREM 3. Let $a, b, c$ be integers relatively prime to $N$. Suppose, for all $k \in \mathbf{Z}-$ $N \mathbf{Z}$,

$$
\langle a k / N\rangle+\langle b k / N\rangle+\langle c k / N\rangle \geqslant 1
$$

Then either

(i) $\langle a k / N\rangle+\langle b k / N\rangle+\langle c k / N\rangle \in \mathbf{Z}$ for all $k$,

(ii) after reordering $a, b, c$, we have $a+b \equiv 0(\bmod N)$, or

(iii) there is some integer $k$ with $(k, N)=1$ such that, after reordering,

$$
(\langle a k / N\rangle,\langle b k / N\rangle,\langle c k / N\rangle)=(1 / 9,4 / 9,7 / 9) \text { or }(1 / 14,9 / 14,11 / 14) \text {. }
$$

Conversely, if either (i), (ii), or (iii) holds, then (*) is true for all $k \in \mathbf{Z}-N \mathbf{Z}$.

Proof. Let $d=-(a+b+c)$. If $d \equiv 0(\bmod N)$ then we have case (i). If $(d, N)=1$ then, for each $k \in \mathbf{Z}-N \mathbf{Z},\langle d k / N\rangle>0$, so that

$$
\langle a k / N\rangle+\langle b k / N\rangle+\langle c k / N\rangle+\langle d k / N\rangle \geqslant 1+\langle d k / N\rangle>1 \text {. }
$$

We may then apply Theorem 2 to conclude that we have case (ii).

Thus, we may assume that $d \not \equiv 0(\bmod N)$ and $(d, N) \neq 1$. In this case, $(a / N, b / N, c / N, d / N)$ is a Fermat quadruple in which exactly three of the numerators are relatively prime to $N$. We apply Theorem 1 to find such Fermat quadruples. In case (i) of Theorem 1, either 0,2 , or 4 of the numerators are relatively prime to $N$, and, in cases (ii) and (iii), at most two of the numerators are relatively prime to $N$. Thus, any Fermat quadruples satisfying our hypothesis will be found in cases (iv) and (v).

Suppose $(1 / 3 K,(K+1) / 3 K,(2 K+1) / 3 K,(3 K-3) / 3 K)$ is a Fermat quadruple (with $N=3 K$ ) from case (iv) of Theorem 1 . In order to satisfy our hypothesis, 1 , $K+1$, and $2 K+1$ must all be relatively prime to $3 K$; in particular, $K \equiv 0(\bmod 3)$. On the other hand,

$$
\langle 3 / 3 K\rangle+\langle 3(K+1) / 3 K\rangle+\langle 3(2 K+1) / 3 K\rangle=3 / K \geqslant 1
$$

so that $K \leqslant 3$. We thus find that the only solution in this case is $K=3$ and $(\langle a k / N\rangle,\langle b k / N\rangle,\langle c k / N\rangle)=(1 / 9,4 / 9,7 / 9)$.

To analyse case (v) of Theorem 1, we have listed in Table 1 all exceptional Fermat quadruples in which exactly three of the numerators are relatively prime to $N$. We 
have also (in all but the first line of the table), listed a value of $k$ and the corresponding triple $(\langle a k / N\rangle,\langle b k / N\rangle,\langle c k / N\rangle)$; since the sum in each case is less than 1 , none of these satisfy our hypothesis. On the other hand, it is easily verified that the remaining case $(1 / 14,9 / 14,11 / 14)$ satisfies the hypothesis for each $k$. Q.E.D.

\begin{tabular}{|c|c|c|}
\hline$(a / N, b / N, c / N, d / N)$ & $k$ & $(\langle a k / N\rangle,\langle b k / N\rangle,\langle c k / N\rangle)$ \\
\hline$(1 / 14,9 / 14,11 / 14,7 / 14)$ & - & -- \\
\hline$(1 / 21,4 / 21,19 / 21,18 / 21)$ & 6 & $(2 / 7,1 / 7,3 / 7)$ \\
\hline$(1 / 28,9 / 28,25 / 28,21 / 28)$ & 7 & $(1 / 4,1 / 4,1 / 4)$ \\
\hline$(1 / 42,31 / 42,37 / 42,15 / 42)$ & 7 & $(1 / 6,1 / 6,1 / 6)$ \\
\hline$(1 / 42,25 / 42,37 / 42,21 / 42)$ & 7 & $(1 / 6,1 / 6,1 / 6)$ \\
\hline$(1 / 78,55 / 78,61 / 78,39 / 78)$ & 13 & $(1 / 6,1 / 6,1 / 6)$ \\
\hline
\end{tabular}

TABLE 1

From Theorem 3, we deduce a classification of isolated canonical cyclic quotient singularities in dimension three. Recall that a cyclic group $G \subset \mathrm{Gl}(3, \mathrm{C})$ of order $N$ gives an isolated singularity $X / G$ exactly when all eigenvalues of a generator $g$ are primitive $N$ th roots of unity.

THEOREM 4. Let $G \subset \mathrm{Gl}(3, \mathrm{C})$ be a cyclic group of order $N$ such that all eigenvalues of a generator $g$ are primitive Nth roots of unity. Then the isolated singularity $X / G$ is canonical if and only if one of the following holds:

(i) $X / G$ is Gorenstein,

(ii) $X / G$ is terminal,

(iii) $G$ is conjugate in $\mathrm{Gl}(3, \mathrm{C})$ to the group generated by

$$
\operatorname{diag}\left(e^{2 \pi i / 9}, e^{8 \pi i / 9}, e^{14 \pi i / 9}\right),
$$

or to that generated by

$$
\operatorname{diag}\left(e^{\pi i / 7}, e^{9 \pi i / 7}, e^{11 \pi i / 7}\right)
$$

Proof. Let $\zeta=e^{2 \pi i / N}$, and choose coordinates so that $g=\operatorname{diag}\left(\zeta^{a}, \zeta^{h}, \zeta^{c}\right)$, with $(a, N)=(b, N)=(c, N)=1$. By the criterion of Reid [9], Shepherd-Barron, and Tai [11], $X / G$ is canonical if and only if

$$
\langle a k / N\rangle+\langle b k / N\rangle+\langle c k / N\rangle \geqslant 1
$$

for all $k \in \mathbf{Z}-N \mathbf{Z}$. On the other hand, by the theorem of Khinich [5] and Watanabe [12], $X / G$ is Gorenstein if and only if det $g=1$ (which holds if and only if $\langle a k / N\rangle+\langle b k / N\rangle+\langle c k / N\rangle \in \mathbf{Z}$ for all $k$ ). Since we proved in [7] that $X / G$ is terminal if and only if case (ii) in Theorem 3 holds, this theorem now follows immediately from Theorem 3. Q.E.D. 


\section{REFERENCES}

1. N. Aoki, On some arithmetic problems related to the Hodge cycles on the Fermat varieties, Math. Ann. 266 (1983), 23-54.

2. N. Aoki and T. Shioda, Generators of the Neron-Severi group of a Fermat surface, Arithmetic and Geometry, Vol. I: Arithmetic, Progress in Math., Vol. 35, Birkhauser, Boston, Basel and Stuttgart, 1983. pp. $1-12$.

3. V. I. Danilov, The birational geometry of toric threefolds, Izv. Akad. Nauk SSSR Ser. Mat. 46 (1982), 972-981 = Math. USSR-Izv. 21 (1983), 269-280.

4. M. A. Frumkin, Description of the elementary three-dimensional polyhedra, First All-Union Conference on Statistical and Discrete Analysis of Non-numerical Information, Expert Estimation and Discrete Optimization (abstract of conference reports), Moscow-Alma-Ata, 1981. (Russian)

5. V. A. Khinich, On the Gorenstein property of the ring of invariants of a Gorenstein ring, Izv. Akad. Nauk SSSR Ser. Mat. 40 (1976), 50-56 = Math. USSR-Izv. 10 (1976), 47-53.

6. W. Meyer and W. Neutsch, Fermatquadrupel, Math. Ann. 256 (1981), 51-62.

7. D. R. Morrison and G. Stevens, Terminal quotient singularities in dimensions three and four, Proc. Amer. Math. Soc. 90 (1984), 15-20.

8. Z. Ran, Cycles on Fermat hypersurfaces, Compositio Math. 42 (1981), 121-142.

9. M. Reid, Canonical 3-folds, Géométrie Algébrique d'Angers (A. Beauville, ed.), Sijthoof and Noordhoff: Alphen aan den Rijn. 1980, pp. 273-310.

10. T. Shioda, On the Picard number of a Fermat surface, J. Fac. Sci. Univ. Tokyo Sect. 1A Math. 28 (1982), 725-734.

11. Y. S. Tai, On the Kodaira dimension of the moduli space of Abelian varieties, Invent. Math. 68 (1982), 425-439.

12. K. Watanabe, Certain invariant subrings are Gorenstein. I, II, Osaka J. Math. 11 (1974), 1-8, 379-388.

13. G. K. White, Lattice tetrahedra, Canad. J. Math. 16 (1964), 389-397.

Department of Mathematics, Princeton University, Princeton, New Jersey 08544

Current address: Research Institute for Mathematical Sciences, Kyoto University, Kitashirakawa, Sakyo-ku, Kyoto 606, Japan 